\title{
VLA POLARIMETRY OF VLBI CORE-JET SOURCES
}

\author{
J.M. Wrobel ${ }^{1}$, T.J. Pearson ${ }^{2}$, M.H. Cohen ${ }^{2}$, and A.C.S. Readhead ${ }^{2}$ \\ ${ }^{1}$ Physics Department, New Mexico Institute of Mining and Technology \\ 2 Owens Valley Radio Observatory, California Institute of Technology
}

Radio imaging of VLBI core-jet sources can be used to examine the case for continuity of jet-like features between parsec and circumgalactic scales. Futhermore, polarimetry of such sources allows investigation of the dominant magnetic field topologies as a function of linear offset from the central engine. Examination of these continuity and field topology issues is essential for an understanding of how energy is channeled from the nuclear regions to the circumgalactic environment.

Linear polarization position angles $\left(\chi_{\lambda}\right)$ in radio images, especially those at wavelengths $\geq$ $18 \mathrm{~cm}$, must be corrected for Faraday rotation before intrinsic magnetic field configurations can be inferred. Rotation measures of VLBI core-jet sources are difficult to determine from broadband (e.g., 2, 6, and $20 \mathrm{~cm}$ ) polarimetry, for two reasons (Rudnick and Jones 1983). First, the wavelength-dependent opacity alters the electron population emitting at different wavelengths. Second, structural complexities and time variations which do not appear simultaneously at all wavelengths make polarization position angle comparisons dubious. Rudnick and Jones (1983) demonstrated that these problems can be overcome by conducting simultaneous, multi-wavelength polarimetry within the $20-\mathrm{cm}$ window of the NRAO VLA.

This technique was applied to 7 VLBI core-jet sources from the survey of Pearson and Readhead (1984). Some properties of these 7 are given in Table I. Snapshots of each source were taken at wavelengths of $21.6,21.2,20.5,19.8,18.3$, and $18.0 \mathrm{~cm}$. The resolution was $\sim 1^{\prime \prime} . \chi_{\lambda}$ is plotted in Figure 1 as a function of $\lambda^{2}$. The data for each source involve measurements either of its unresolved VLA core (size $<1^{\prime \prime}$ or $\leq 4 \mathrm{kpc}-$ see col. 4), or of its kpc-scale emission adjacent to the VLA core. For each source, the data were fit to a straight line by the method of least squares. Each fit yielded a zero-wavelength intercept $\left(\chi_{0}\right.$, col. 5$)$ and a rotation measure (RM, col. 6$)$.

The rotation measures resemble those of nine $3 \mathrm{C}$ sources with similar ranges of Galactic longitude and latitude $\left(\mathrm{l}^{I I}=75-115^{\circ}\right.$ and $\mathrm{b}^{I I}=20-50^{\circ}$; Simard-Normandin, Kronberg, and Button 1981). This is consistent with a primarily Galactic origin for the rotation measures of both the (presumably steep-spectrum) 3C sources and the VLBI core-jet sources. If the Galactic disk behaves like an unresolved foreground screen, then it should cause Faraday rotation but no depolarization (Laing 1984). Such behavior is shown by $1803+784$, the core-jet source with the largest rotation, for which the observed percent polarization $p_{\lambda}$ is remarkably constant at 3.9 percent between 21.6 and $18 \mathrm{~cm}$. For comparison, if the RM was due to mixed thermal and emitting plasma, then a simple uniform slab model would predict a factor of 5 drop in $p_{\lambda}$ between 21.6 and $18 \mathrm{~cm}$.

For 5 sources $\chi_{0}$ refers to the unresolved VLA core and can be compared to the VLBI structural position angle $\chi_{v}$ at $6 \mathrm{~cm}$ for $1642+690$ (Pearson et al. 1986) and at $18 \mathrm{~cm}$ for $0836+710,1749+701$, $1803+784$, and $1928+738$ (Eckart et al. 1987). Col. 7 gives the value of the acute angle A between $\chi_{0}$ and $\chi_{v}$. For sources with multiple VLBI components, $\chi_{v}$ refers to the outermost one. If the linearly polarized emission is due to optically thin synchrotron emission, then $\mathrm{A} \geq 75^{\circ}(0836+710$, $1642+690,1928+738)$ indicates a projected magnetic field fairly well aligned with the VLBI jet. Bridle (1984) finds a similar trend among kpc-scale jets that, like the VLBI jets, are one-sided. The small A values found for $1749+701$ and $1803+784$ could be caused by, for example, the polarized emission originating in regions whose structural angles differ from those of the VLBI components; or else in regions with similar structural angles but whose magnetic fields are associated with shocks in the jet. 


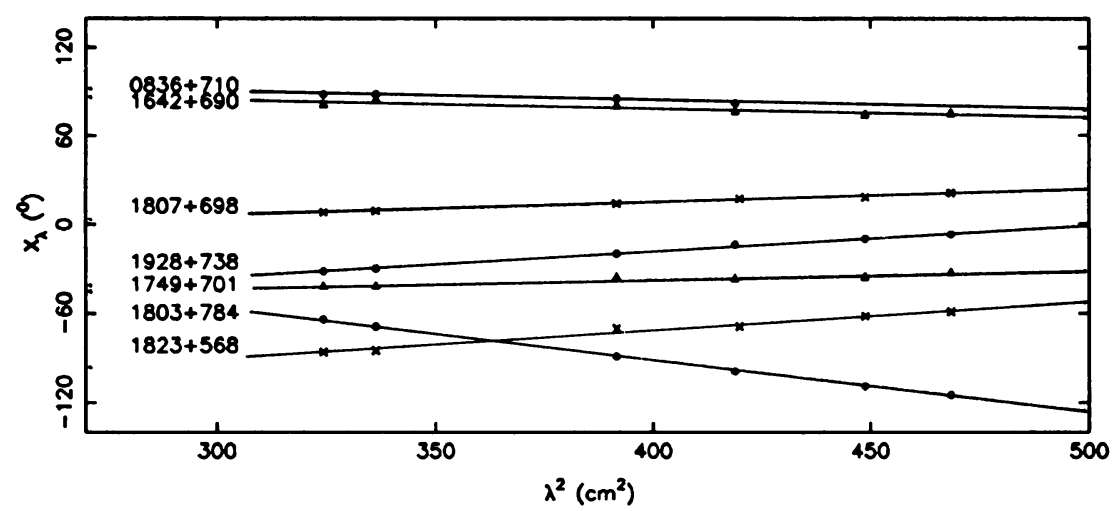

Fig. 1. - Linear polarization position angle $\chi_{\lambda}$ as a function of $\lambda^{2}$. Symbols distinguish between polarimetry of an unresolved VLA core $\left(<1^{\prime \prime}\right.$, filled symbols) and of adjacent kpc-scale emission (crosses). Straight lines are the results of least-squares fits.

\section{References}

Bridle, A.H.: 1984, Astr. J. 89, 979-986.

Eckart, A., et al.: 1987, Astr. Ap. Suppl. 67, 121-146.

Laing, R.A.: 1984, in Physics of Energy Transport in Extragalactic Radio Sources, ed. A.H. Bridle and J.A. Eilek (NRAO: Greenbank), pp. 90-96.

Lawrence, C.R., et al.: 1986, Astr. J. 91, 494-495.

Pearson, T.J., and Readhead, A.C.S.: 1984, in IAU Symposium 110, VLBI and Compact Radio Sources, ed. R. Fanti, K. Kellermann, and G. Setti (Dordrecht: Reidel), pp. 15-24.

Pearson, T.J., et al.: 1986, Ap. J. Letters 300, L25-L29.

Rudnick, L. and Jones, T.W.: 1983, Astr. J. 88, 518-526.

Simard-Normandin, M., Kronberg, P.P., and Button, S.: 1981, Astroph. J. Suppl. 45, 97-111.

Wrobel, J.M.: 1987, in Superluminal Radio Sources, ed. J.A. Zensus and T.J. Pearson (Cambridge Univ. Press: Cambridge), pp. 186-192.

Table I. Seven VLBI Core-Jet Sources

\begin{tabular}{ccccccc}
\hline \hline IAU & Common & $\mathrm{Id}^{a}$ & $\begin{array}{c}\mathrm{Scale}^{b} \\
\left(\mathrm{kpc} /{ }^{\prime \prime}\right)\end{array}$ & $\begin{array}{c}\chi_{0} \\
\left({ }^{0}\right)\end{array}$ & $\begin{array}{c}\mathrm{RM} \\
\left(\mathrm{rad} \mathrm{m}^{-2}\right)\end{array}$ & $\begin{array}{c}\mathrm{A} \\
\left({ }^{0}\right)\end{array}$ \\
\hline $0836+710$ & $4 \mathrm{C} 71.07$ & $\mathrm{Q}$ & 4.0 & $+109 \pm 4$ & $-11 \pm 2$ & 75 \\
$1642+690$ & $4 \mathrm{C} 69.21$ & $\mathrm{Q}$ & 4.1 & $+103 \pm 5$ & $-11 \pm 2$ & 88 \\
$1749+701$ & $\ldots$ & $\mathrm{B}$ & 4.1 & $-61 \pm 4$ & $+10 \pm 2$ & 16 \\
$1803+784$ & $\ldots$ & $\mathrm{B}$ & 4.0 & $+51 \pm 2$ & $-62 \pm 1$ & 30 \\
$1807+698$ & $3 \mathrm{C} 371$ & $\mathrm{G}$ & 0.7 & $-20 \pm 2$ & $+15 \pm 1^{c}$ & $\ldots$ \\
$1823+568$ & $4 \mathrm{C} 56.27$ & $\mathrm{~B}$ & 3.9 & $-148 \pm 6$ & $+33 \pm 2$ & $\ldots$ \\
$1928+738$ & $4 \mathrm{C} 73.18$ & $\mathrm{Q}$ & 2.7 & $-89 \pm 2$ & $+31 \pm 1$ & 83 \\
\hline
\end{tabular}

Notes -

a $\mathrm{Q}=$ quasar, $\mathrm{B}=$ BL Lac object, $\mathrm{G}=$ galaxy.

${ }^{b} \mathrm{H}_{0}=100 \mathrm{~km} \mathrm{~s}^{-1} \mathrm{Mpc}^{-1}$ and $\mathrm{q}_{0}=0.5$. Redshifts are from Pearson and Readhead 1984, Lawrence et al. 1986, Eckart et al. 1987, and Lawrence, private communication.

c Preliminary RM given by Wrobel 1987. 